\title{
Temperature dependence of universal conductance fluctuations in narrow mesoscopic Si inversion layers
}

\author{
J. R. Gao, J. Caro, A. H. Verbruggen, and S. Radelaar \\ Delft Institute for Microelectronics and Submicron Technology, Delft University of Technology, \\ Delft, The Netherlands \\ J. Middelhoek \\ Vakgroep IC-Technology and Electronics, Twente University, Enschede, The Netherlands
}

(Received 21 July 1989)

\begin{abstract}
The temperature dependence of magnetoconductance fluctuations in narrow mesoscopic silicon inversion layers (length $4.3 \mu \mathrm{m}$, width 0.14 and $0.43 \mu \mathrm{m}$ ) has been studied. The magnitude and magnetic correlation length of the fluctuations was measured in the temperature range $T=0.2-4.2 \mathrm{~K}$ and for magnetic fields up to $3 \mathrm{~T}$. Weak-localization experiments were performed for an independent determination of the inelastic diffusion length $L_{\text {in }}$. When $L_{\text {in }}$ exceeds the channel width $W$ [quasi-one-dimensional (quasi-1D)] the experimental temperature dependence of the fluctuation amplitude and magnetic correlation length is very well described by the theory for universal conductance fluctuations of Lee and Stone. In case $L_{\text {in }}<W$ (quasi-2D) a good agreement with this theory is found for the temperature dependence of the fluctuation amplitude.
\end{abstract}

\section{INTRODUCTION}

It is well established now both by experimental ${ }^{1}$ and theoretical $^{2}$ investigations that mesoscopic normal-metal wires and rings exhibit random, reproducible fluctuations in the magnetoconductance. These fluctuations are manifestations of quantum interference of electron waves in coherent sample sections of linear size $L_{\text {in }}$, the inelastic diffusion length of the electrons. They are called universal conductance fluctuations (UCF) because at zero temperature their magnitude expressed as the root-meansquare deviation from the mean is of order of $e^{2} / h$, regardless of degree of disorder and sample size. The shape of the fluctuation pattern is sensitive to the microscopic distribution of elastic scattering centers for the electrons and for this reason the fluctuation traces are sometimes referred to as magnetofingerprints. At finite temperature the size of the sample usually exceeds the inelastic diffusion length and due to classical self-averaging the fluctuations are reduced by a factor determined by the ratio of the sample size to the inelastic diffusion length.

Among the systems used for UCF studies, inversion and accumulation layers in Si metal-oxide-semiconductor field-effect transistors (MOSFET's) play an important role, since they offer the additional possibility of varying the Fermi energy by varying the gate voltage. Aperiodic magnetoconductance fluctuations in narrow $\mathrm{Si}$ inversion layers were reported for the first time by Licini et al. ${ }^{3}$ Only a preliminary comparison of the data was made with the (at that time) very recent theory of Lee and Stone. $^{2}$ Later Kaplan and Hartstein ${ }^{4}$ showed that in pinched accumulation layers magnetoconductance fluctuations only depend on the normal component of the magnetic field, demonstrating the Aharonov-Bohm effect origin of the fluctuations. Skocpol et al. ${ }^{5}$ demonstrated that experimental fluctuation amplitudes are in good agreement with theoretical predictions for a wide range of the ratio of the inelastic length to the sample size. However, the data presented is limited to two temperatures only ( $T=2 \mathrm{~K}$ and $T=4.2 \mathrm{~K}$ ).

According to the theory of Lee and Stone ${ }^{2}$ the temperature dependence of the amplitude and the magnetic correlation length of the fluctuations is determined by the temperature dependence of the inelastic diffusion length of the electrons. Thus, by varying the temperature of the sample an important and straightforward experimental test of the theory can be made. Detailed checks of theoretical predictions for the temperature dependence of UCF have been carried out in small metal loops ${ }^{6}$ and split gate heterojunction FET's. ${ }^{7}$ So far, however, such detailed experiments on small Si MOSFET's have not been reported.

In this paper we present new experimental data on universal conductance fluctuations in narrow-channel MOSFET's. The dimensions of the inversion channels are $L=4.3 \mu \mathrm{m}$ and $W=0.14$ and $0.43 \mu \mathrm{m}$. The emphasis of the work is on the temperature dependence of universal conductance fluctuations in the magnetoconductance.

This paper is organized as follows. The theoretical relations necessary for the interpretation of the experiments are presented in Sec. II. The fabrication of the devices and the experimental technique are summarized in Sec. III. In addition to experiments on magnetoconductance fluctuations, Sec. IV also describes weak localization measurements at low magnetic fields that were used for an independent determination of the inelastic diffusion length of the electrons. The conclusions of this work are summarized in Sec. V. 


\section{TEMPERATURE DEPENDENCE OF UNIVERSAL CONDUCTANCE FLUCTUATIONS}

The experimental results on universal conductance fiuctuations presented below will be discussed in terms of the root-mean-square ( $\mathrm{rms}$ ) magnitude of the fluctuations and the magnetic correlation length and their temperature dependences. The rms magnitude of the fluctuations is defined as

$$
\operatorname{rms}(g)=\left\langle(g-\langle g\rangle)^{2}\right\rangle^{1 / 2}=\left\langle\delta g^{2}\right\rangle^{1 / 2} .
$$

Here $g=G /\left(e^{2} / h\right)$ is the reduced conductance. In Eq. (1) and in Eq. (2) below, the angular brackets denote averaging over magnetic field. The magnetic correlation length $B_{c}$ can be viewed ${ }^{2}$ as a typical field range beyond which the phases of interference paths contributing to the fluctuation pattern in the conductance become uncorrelated with the phases at the initial field and it is a typical scale of the spacing between peaks and valleys in $g$ as function of $B$. The magnetic correlation length is obtained from the half-width of the corresponding correlation function in magnetic field, defined as

$$
F(\Delta B)=\langle\delta g(B) \delta g(B+\Delta B)\rangle .
$$

The temperature dependence of $\operatorname{rms}(g)$ and $B_{c}$ depends on the size of the conductor in relation to the temperature dependent cutoff lengths $L_{\text {in }}$ and $L_{T}$. The inelastic diffusion length $L_{\text {in }}$ is defined as $\left(D \tau_{\text {in }}\right)^{1 / 2}, D$ being the electron diffusion constant and $\tau_{\text {in }}$ the inelastic scattering time, while the thermal length $L_{T}$ is given by $(h D / k T)^{1 / 2}$. In case $L_{\text {in }}<L_{T}<L$ (which applies to our experiments) $\mathrm{rms}(g)$ of the magnetoconductance fiuctuations for the $1 \mathrm{D}$ and $2 \mathrm{D}$ geometry is given by ${ }^{2}$

$$
\begin{aligned}
& \operatorname{rms}(g)=0.52\left[\frac{L_{\text {in }}}{L}\right]^{3 / 2}, L_{\text {in }}>W \\
& \operatorname{rms}(g)=0.61\left(\frac{W}{L_{\text {in }}}\right)^{1 / 2}\left[\frac{L_{\text {in }}}{L}\right]^{3 / 2}, L_{\text {in }}<W .
\end{aligned}
$$

The magnetic correlation length $B_{c}$ for the 1D and 2D geometry is given by ${ }^{2}$

$$
\begin{aligned}
& B_{c}=1.2 \frac{h}{e} \frac{1}{W L_{\mathrm{in}}}, \quad L_{\mathrm{in}}>W \\
& B_{c}=1.2 \frac{h}{e} \frac{1}{L_{\mathrm{in}}^{2}}, \quad L_{\mathrm{in}}<W .
\end{aligned}
$$

The temperature dependence of $\operatorname{rms}(g)$ and $B_{c}$ in these equations arises from the dependence of $L_{\text {in }}$ on $\tau_{\text {in }}$, which is normally assumed to be proportional to $T^{p}$. The exponent $p$ is characteristic for the inelastic-scattering process that is operative.

\section{DEVICES AND EXPERIMENTAL TECHNIQUE}

The devices used for the present study are $n$-channel $\mathrm{Si}$ MOSFET's with a narrow gate. The gate length $L$ is 4.3 $\mu \mathrm{m}$ and the gate width $W$ is 0.14 and $4.3 \mu \mathrm{m}$. The substrates are $2^{\prime \prime} p$-type silicon wafers with a resistivity of

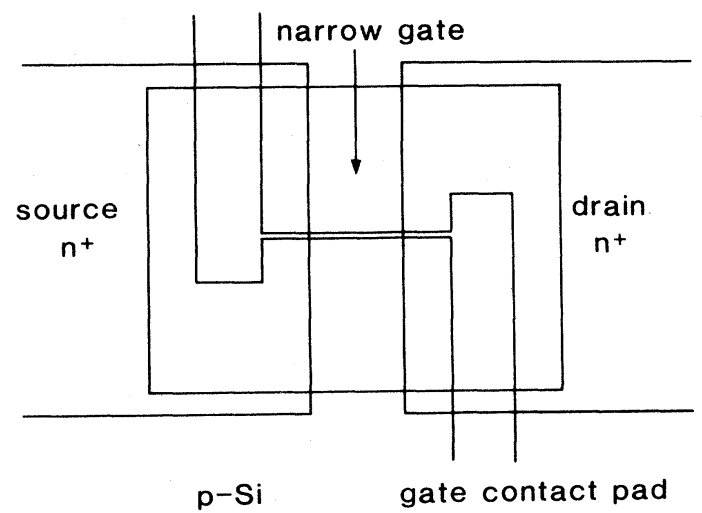

FIG. 1. Schematic layout of narrow channel Si MOSFET.

15-20 $\Omega \mathrm{cm}$. The large structures of the devices (source, drain, etc.) are defined by optical lithography, while the narrow gates and their contact pads are patterned by electron beam lithography. After the growth of a 25$\mathrm{nm}$-thick gate oxide the gate pattern is written in a double layer of polymethyl methacrylate (PMMA), resists with different molecular weight. Then nickel-chromium (NiCr) gates are produced by evaporation and liftoff. In a similar $e$-beam fabrication step $\mathrm{NiCr}$ intermediate contact pads to the narrow gates are formed. The chemicalvapor deposition oxide deposited subsequently protects the narrow lines during the damage annealing and enables the simultaneous contact hole etching to the $\mathrm{NiCr}$ pads and to the source and drain areas. Source and drain each have two aluminum contacts to allow for four terminal measurements. More details of the fabrication process can be found elsewhere. ${ }^{8}$ We fabricated several types of devices with different $W / L$ ratios, the smallest gate width realized being $140 \mathrm{~nm}$. At helium temperatures the devices are normally off and the maximum mobility of the narrowest devices is about $8000 \mathrm{~cm}^{2} / \mathrm{V} \mathrm{s}$. Figure 1 shows a schematic layout of a narrow channel MOSFET fabricated according to this process. The figure shows the source and drain regions and in the center the rectangular area of the gate oxide with the narrow gate and contact pads.

In several types of experiments on different devices the conductance of the inversion channel has been measured in the temperature range between 0.2 and $4.2 \mathrm{~K}$. The measurements were carried out in a ${ }^{3} \mathrm{He}-{ }^{4} \mathrm{He}$ dilution refrigerator equipped with a superconducting magnet. For the conductance measurements a lock-in technique was used. The device was excited by a low-frequency current and the voltage across the channel was kept below $k T / e$ in order to avoid electron heating.

\section{EXPERIMENTAL RESULTS}

\section{A. Weak localization and high-field magnetoconductance}

In Figs. 2 and 3 results are given of low- and high-field magnetoconductance measurements and of measurements of the temperature dependence of the conductance. We carried out such experiments on a number of devices also used for UCF measurements, in order to determine 
the inelastic diffusion length and the dimensionality.

Figure 2(a) shows a plot of the inelastic length in device $N 7$ as a function of $T^{-1 / 2}$. This device has a 0.14 $\mu \mathrm{m}$-wide gate. The values of $L_{\text {in }}$ were derived from fits of the theoretical $1 \mathrm{D}$ expression ${ }^{9}$ for the low-field magnetoconductance (LFMC) to the experimental data. The width of the channel was also obtained as a result of the fits and agreed within five percent with the lithographic width of the gate. The experimental data for temperatures between 1.76 and $4.2 \mathrm{~K}$ and the corresponding fits are shown in the inset of Fig. 2(a). As may be seen the fits describe the experimental data very well. We conclude that the MOSFET is one dimensional with respect to weak localization, although marginally at $4.2 \mathrm{~K}$. Further, $L_{\text {in }}$ depends linearly on $T^{-1 / 2}$, the best fit for the experimental conditions of Fig. 2(a) being $L_{\text {in }}=0.3$ $[T /(1 \mathrm{~K})]^{-1 / 2} \mu \mathrm{m}$. Apparently in our devices $\tau_{\text {in }} \propto L_{\text {in }}^{2}$ is proportional to $1 / T$. This indicates ${ }^{10}$ that electronelectron interaction is the dominant inelastic-scattering mechanism.

In studying fluctuations in the magnetoconductance we
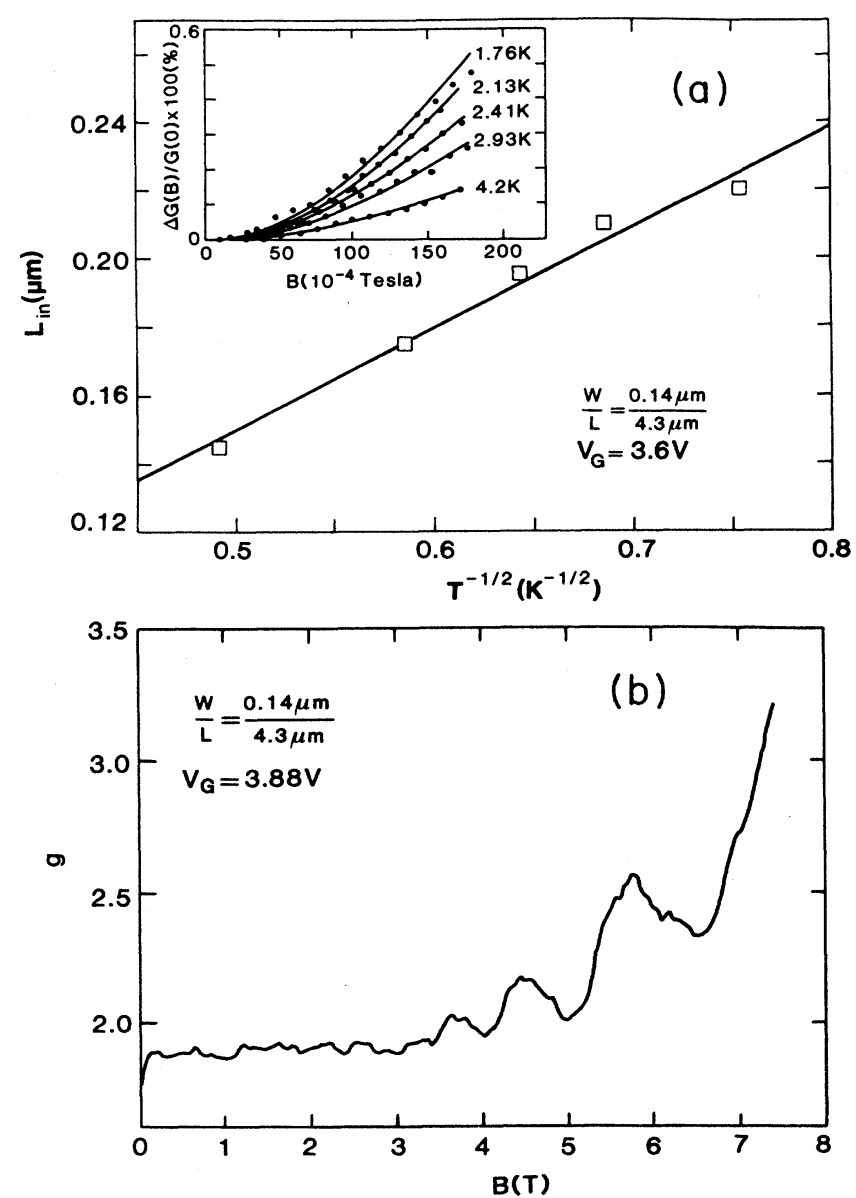

FIG. 2. In (a) the inelastic length $L_{\text {in }}$ in narrow device $N 7$ obtained from the LFMC is plotted against $T^{-1 / 2} . V_{G}=3.6 \mathrm{~V}$. Magnetoconductance data and fits are displayed in inset. For the same device (b) shows SdH oscillations above $3 \mathrm{~T}$ and UCF below $3 \mathrm{~T}$. $T=0.25 \mathrm{~K}$. were limited to magnetic fields up to about $3 \mathrm{~T}$, since above this value at temperatures below $4.2 \mathrm{~K}$ the magnetoconductance is dominated by the Shubnikov-de Haas (SdH) effect. Figure 2(b) gives a magnetoconductance curve of device $N 7$ that clearly exhibits the SdH effect at high fields, reflecting the high mobility of the MOSFET, while for $B<3 \mathrm{~T}$ the trace only shows aperiodic fluctuations.

We generally found that the electron density $N_{s}$ deduced from the $\mathrm{SdH}$ effect agrees well with the value of $N_{s}$ derived from the MOS-capacitor formula. For instance, for the experimental situation of Fig. 2(b) the SdH effect gives $N_{s}=2.1 \times 10^{12} \mathrm{~cm}^{-2}$, while the MOScapacitor formula yields $N_{s}=2.2 \times 10^{12} \mathrm{~cm}^{-2}$. Further, a plot of the Landau index versus inverse magnetic field shows good linearity, indicating ${ }^{11}$ that effects due to $1 \mathrm{D}$ subbands play no role here.

Figure 3(a) shows the conductance in zero magnetic field of device $N 7$ as a function of $T^{-1 / 2}$, for two gate voltages, the temperature ranging form 0.3 to $2.1 \mathrm{~K}$. The lines in Fig. 3(a) are linear fits to the data points. We an-
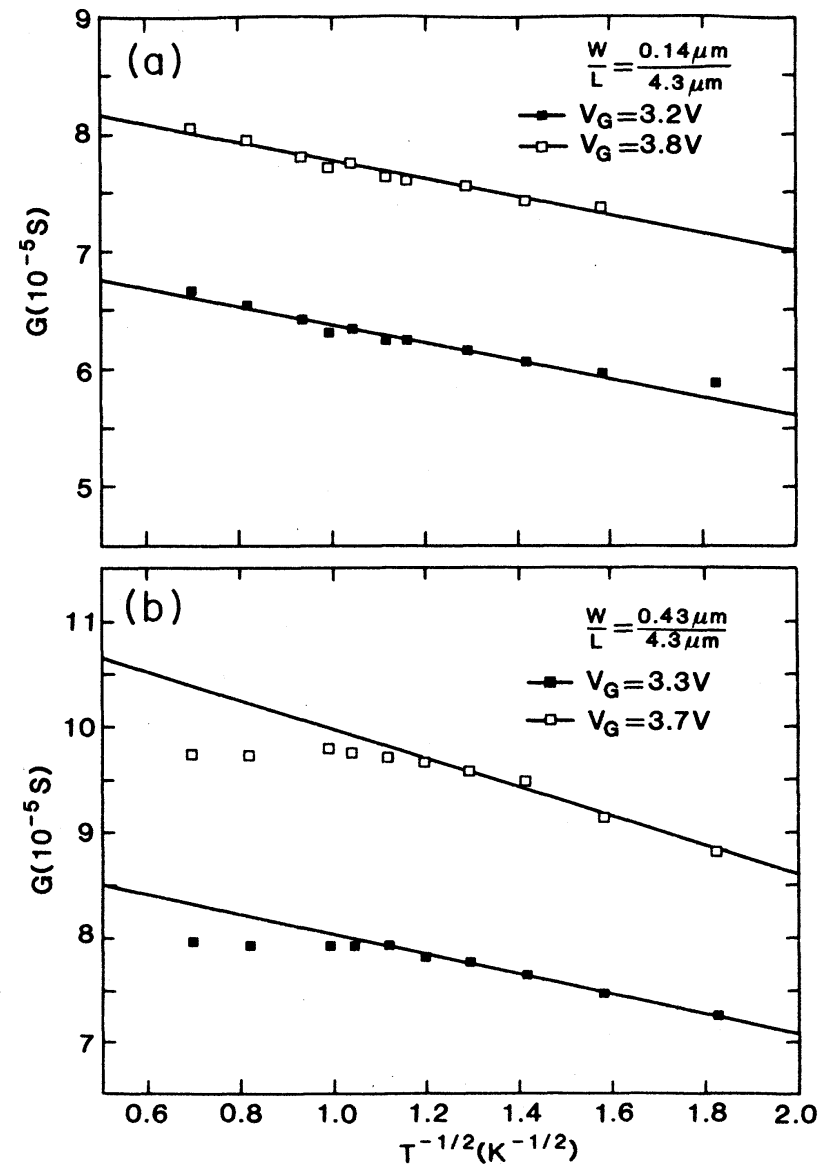

FIG. 3. In (a) the conductance of device $N 7$ is plotted against $T^{-1 / 2}$ in zero magnetic field for two gate voltages. The temperature ranges from 0.3 to $2.1 \mathrm{~K}$. (b) shows the conductance of "wide" device $N 19$ plotted against $T^{-1 / 2}$ for two gate voltages. Straight lines are linear fits to the low-temperature data points and serve to guide the eye. 
alyzed these data in a way similar to that of Skocpol et $a l .{ }^{12}$ and treated the correction to the Boltzmann conductance $\Delta \boldsymbol{G}$ of the one-dimensional inversion channel as an addition of a weak localization term and an electronelectron interaction term:

$$
\Delta G=-\frac{e^{2}}{\pi \hbar L}\left[L_{\mathrm{in}}+\frac{0.868}{\pi \sqrt{\pi}}\left[4-\frac{3}{2} F_{\sigma}\right] L_{T}\right]
$$

Here $F_{\sigma}=32\left[1+F / 4-(1+F / 2)^{1 / 2}\right] / F$ and $F$ is a screening parameter. ${ }^{13}$ This approach is correct for the temperature range of Fig. 3(a), since both $L_{\text {in }}$ and $L_{T}$ exceed the width of the channel. To evaluate $\Delta G$ we extrapolated the inelastic lengths from our magnetoconductance data [Fig. 2(a)] to lower temperatures and scaled these for different conductance values, using $L_{\text {in }} \propto D \propto G$ (which follows ${ }^{12}$ from $\tau_{\text {in }} \propto D$ ). The two-dimensional diffusion coefficient was calculated from $D=G_{\square} / e^{2} N_{2}$, where $N_{2}$ is the two-dimensional density of states. $G_{\square}$ was determined at a temperature high enough for $\Delta G$ as given by Eq. (5) to be negligible. The same values of $D$ served to calculate $L_{T}$. The screening parameter $F$ was set to unity. From the analysis we found that the contributions of weak localization and electron-electron interaction effects are comparable in magnitude. Further, using the experimental values for $L_{\text {in }}$ and $L_{T}$ in Eq. (5) we obtained values for the slope of the lines in Fig. 3(a). These values are $-10.3 \mu \mathrm{S} \mathrm{K}^{1 / 2}\left(V_{G}=3.2 \mathrm{~V}\right)$ and -11.9 $\mu \mathrm{S} \mathrm{K}^{1 / 2}\left(V_{G}=3.8 \mathrm{~V}\right)$, to be compared with experimental values of $-7.7 \mu \mathrm{S} \mathrm{K}^{1 / 2}$ and $-8.0 \mu \mathrm{S} \mathrm{K}^{1 / 2}$, respectively. We conclude, taking into account the uncertainties in deriving the semitheoretical values ( $F$ parameter, extrapolation inelastic length) that there is good agreement. Also, the linearity of the $G-T^{-1 / 2}$ plot confirms the $1 / T$ dependence of $\tau_{\text {in }}$ as found from the LFMC.

The temperature dependence of the conductance of device $N 19$, which has a $0.43-\mu \mathrm{m}$-wide gate, is given in Fig. 3(b) for two gate voltages. The data points suggest that the temperature dependence can be divided in two regimes. Below $0.9 \mathrm{~K}$ the conductance is approximately linearly decreasing with increasing $T^{-1 / 2}$, but above this transition point the conductance is almost constant. LFMC traces taken from this device can be well fitted by the theoretical 2D expression ${ }^{10}$ and yield $L_{\text {in }}(0.3$ $\mathrm{K})=0.31 \mu \mathrm{m}$. Thus, for the temperature range of Fig. 3(b) $(0.3-2.1 \mathrm{~K})$ the device is two dimensional with respect to weak localization. On the other hand, at $0.9 \mathrm{~K}$ $L_{T}$ for the two curves is approximately equal to the width. From this we conclude that at the transition point a dimensional crossover takes place for electron-electron interaction effects. The approximately constant conductance level above $0.9 \mathrm{~K}$ can be understood as a compensation of the classical Boltzmann conductance and the contributions to the conductance of 2D weak localization and $2 \mathrm{D}$ interaction effects. This picture is supported by a reasonable agreement of the slope of the linear part of the experimental lines and the values calculated from the second term of Eq. (5).

\section{B. Universal conductance fluctuations}

Traces of fluctuations in the magnetoconductance of device $N 25(W / L=0.14 \mu m / 4.3 \mu \mathrm{m})$ are shown in Fig. 4 , in the field range between 0 and $3 \mathrm{~T}$ and for temperatures between 0.2 and $4.2 \mathrm{~K}$. The gate voltage was $4.0 \mathrm{~V}$, giving an electron density of $3.4 \times 10^{12} \mathrm{~cm}^{-2}$. As may clearly be seen from the figure the amplitude of the fluctuations gradually decreases with increasing temperature. At $4.2 \mathrm{~K}$ the fluctuations are small but still visible. In several experiments the magnetic field was swept across its zero value. The resulting traces were symmetric as expected for our two probe structure. Figure 4 shows two traces taken at $0.5 \mathrm{~K}$. These were obtained under identical experimental conditions. The lower one was obtained first and the time interval between the measurements was $15 \mathrm{~h}$. During this time the sample was kept below $1 \mathrm{~K}$ and other measurements were performed. Apparently there is an excellent reproducibility of the fluctuations in this device. To demonstrate the gate voltage dependence of the magnetoconductance Fig. 5 shows traces taken from the same device, in the same experimental run, but at a gate voltage of $4.2 \mathrm{~V}$. In comparing Figs. 4 and 5 one concludes that the two magnetofingerprints are completely different, which is due to different interference conditions at a different Fermi energy.

In order to calculate $\operatorname{rms}(g)$ as a function of temperature a background was subtracted from each curve that was obtained from a polynomial fit to the $4.2 \mathrm{~K}$ curve $\left(V_{G}=4.0 \mathrm{~V}\right)$ or the $1.54 \mathrm{~K}$ curve $\left(V_{G}=4.2 \mathrm{~V}\right)$. To eliminate the influence of weak localization, calculations were limited to $B>0.4 \mathrm{~T}$. This procedure was followed for two data sets that include the curves of Figs. 4 and 5. For $V_{G}=4.0 \mathrm{~V}$ the $4.2 \mathrm{~K}$ data point was omitted, since

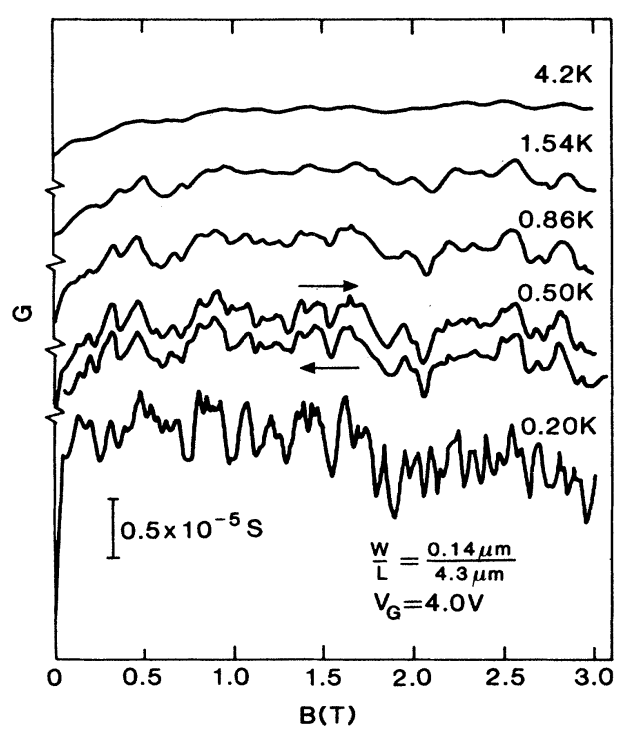

FIG. 4. Universal conductance fluctuations in the magnetoconductance of narrow device $N 25$ for a number of temperatures. $V_{G}=4.0 \mathrm{~V}$. The time interval between the two traces at $0.5 \mathrm{~K}$ was $15 \mathrm{~h}$ and they were taken with opposite sweep direction. For each of the six curves $\langle G\rangle$ amounts to about $108 \mu \mathrm{S}$. 


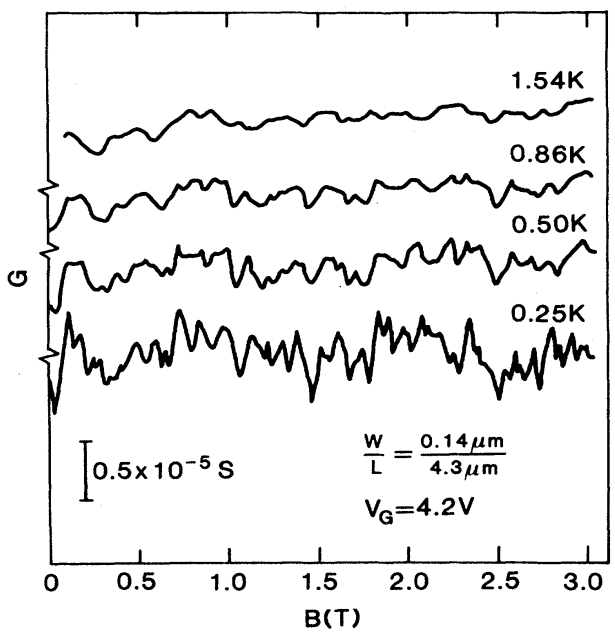

FIG. 5. Universal conductance fluctuations in the magnetoconductance of narrow device $N 25$ for a number of temperatures. $V_{G}=4.2 \mathrm{~V}$. For each of the four curves $\langle G\rangle$ amounts to about $113 \mu \mathrm{S}$.

from the LFMC experiment at $1 \mathrm{~K}$ discussed below, we found that at $4.2 \mathrm{~K}$ the device is not in the onedimensional limit. For the temperature range $0.2-1.54$ $\mathrm{K}$ results are plotted in Fig. 6. The straight lines in Fig. 6 are least-square fits to the data points. For $V_{G}=4.0 \mathrm{~V}$ the best fit is $\mathrm{rms}(g)^{\text {expt }}=(2.7 \pm 0.2) \times 10^{-2} \quad[T / 1$ $\mathrm{K})]^{-0.63 \pm 0.06}$ while for $V_{G}=4.2 \mathrm{~V}$ we find $\operatorname{rms}(g)^{\operatorname{expt}}$ $=(1.7 \pm 0.1) \times 10^{-2}[T /(1 \mathrm{~K})]^{-0.61 \pm 0.07}$.

In order to compare these results with the theory of the universal conductance fluctuations, ${ }^{2}$ we first determined the dimensionality of the device from the inelastic length and the thermal length. The inelastic length was calculated from the low-field magnetoconductance at $V_{G}=4.0 \mathrm{~V}$, which for this device was measured only at $1.0 \mathrm{~K}$. The result is $L_{\text {in }}(1 \mathrm{~K})=(0.29 \pm 0.06) \mu \mathrm{m}$. Using

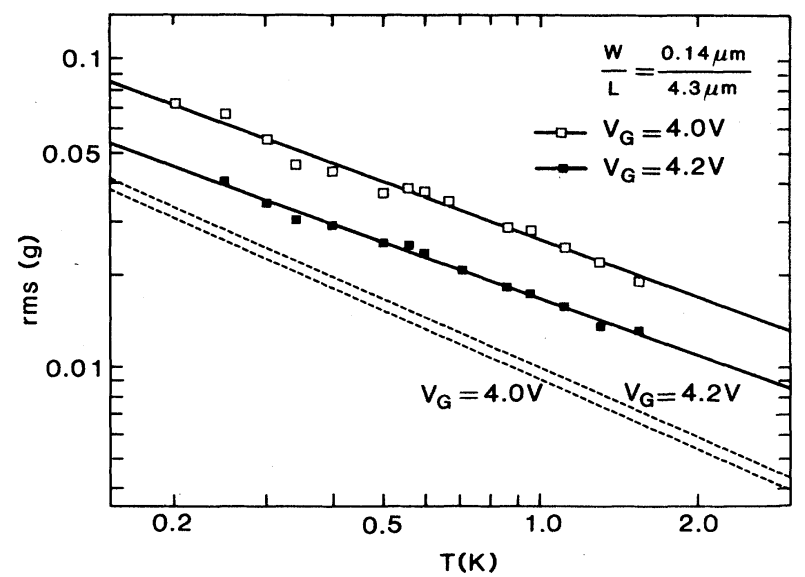

FIG. 6. Temperature dependence of $\operatorname{rms}(g)$ of the fluctuations on a $\log -\log$ scale for $V_{G}=4.0 \mathrm{~V}$ and $V_{G}=4.2 \mathrm{~V}$. Dashed lines are theoretical relations corresponding to the experimental data. the $T^{-1 / 2}$ behavior found for other devices we find $L_{\text {in }}(T=1.54 \mathrm{~K})=0.23 \mu \mathrm{m}$. The thermal length was calculated from the electron diffusion constant derived from the average conductance $\langle G\rangle$ at $V_{G}=4.0 \mathrm{~V}$, which is constant to within a few percent in the temperature range $0.2-4.2 \mathrm{~K}$ and amounts to $108 \mu \mathrm{S}$. In this way it was found that $L_{T}(1.54 \mathrm{~K})=0.63 \mu \mathrm{m}$. Since $L_{T}(1.54)>W$ and $L_{\text {in }}(1.54)>W$ the device is one dimensional for the temperature range of Fig. 6. This holds for $V_{G}=4.2 \mathrm{~V}$ as well, since $L_{\text {in }} \propto g$ and $L_{T} \propto g^{1 / 2}$. Now, by using the values for $L_{\text {in }}$ and $W$ derived from the LFMC measurements, Eq. (3a) predicts $\mathrm{rms}(g)^{\text {theor }}=9.1 \times 1^{-3}[T /(1$ $\mathrm{K})]^{-0.75}$ and $\mathrm{rms}(\mathrm{g})^{\text {theor }}=9.8 \times 10^{-3}[T /(1 \mathrm{~K})]^{-0.75}$, for $V_{G}=4.0 \mathrm{~V}$ and $V_{G}=4.2 \mathrm{~V}$, respectively. Both theoretical relations are given as dashed lines in Fig. 6. Since $\operatorname{rms}(g)$ for the higher gate voltage has the larger $L_{\text {in }}$, the theoretical $\operatorname{rms}(g)$ for $V_{G}=4.2 \mathrm{~V}$ is above that for $V_{G}=4.0 \mathrm{~V}$. For the experimental lines this is just the other way around. At present we have no explanation for the fact that experimentally $\mathrm{rms}(g)$ at $4.0 \mathrm{~V}$ exceeds $\operatorname{rms}(g)$ at $4.2 \mathrm{~V}$. However, the magnitudes of $\operatorname{rms}(g)^{\text {expt }}$ and $\operatorname{rms}(g)^{\text {theor }}$ in Fig. 6 are in good agreement. Further, the slopes of the experimental lines are strongly indicative for a $T^{-1 / 2}$ behavior of $L_{\text {in }}$.

On the two sets of UCF data a further analysis was carried out to yield the magnetic correlation lengths. We note that the magnetic field range in the experiment is not large enough to observe several "periods" of the slow fluctuations in the traces of Fig. 4. Therefore in calculating the correlation functions of the traces taken at $4.0 \mathrm{~V}$ we fitted a linear function to each trace and used it as a background. This is necessary, since if not done the correlation functions are severely disturbed. For reasons of consistency the same procedure was followed for the traces taken at $4.2 \mathrm{~V}$. For $V_{G}=4.0 \mathrm{~V}$ obtained correlation functions are displayed in Fig. 7. The correlation functions decay smoothly to zero. In Fig. 8 resulting correlation lengths $B_{c}$ are plotted as a function of temperature. The lines are best fits and are given by $B_{c}^{\text {expt }}=(74 \pm 5)[T /(1 \mathrm{~K})]^{0.56 \pm 0.07} \mathrm{mT}$ and $B_{c}^{\text {expt }}=(48 \pm 3)$

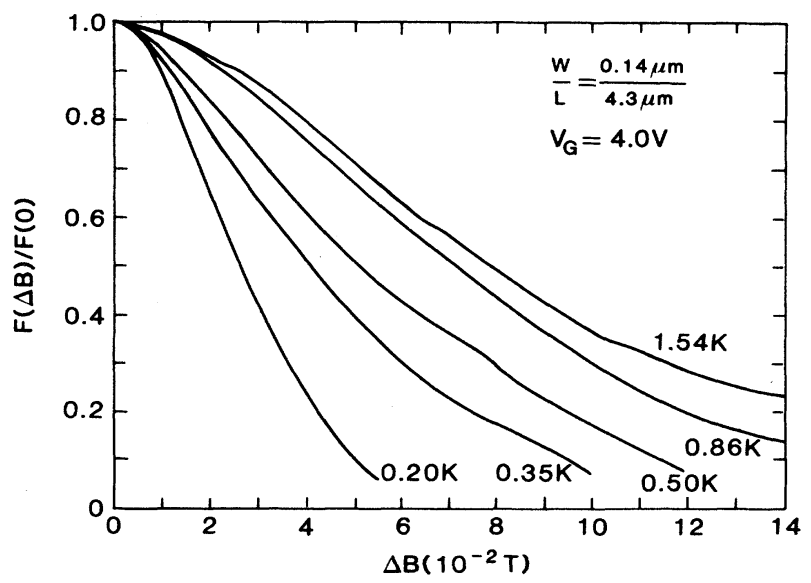

FIG. 7. Normalized correlation functions in magnetic field for fluctuation traces taken at $V_{G}=4.0 \mathrm{~V}$. 


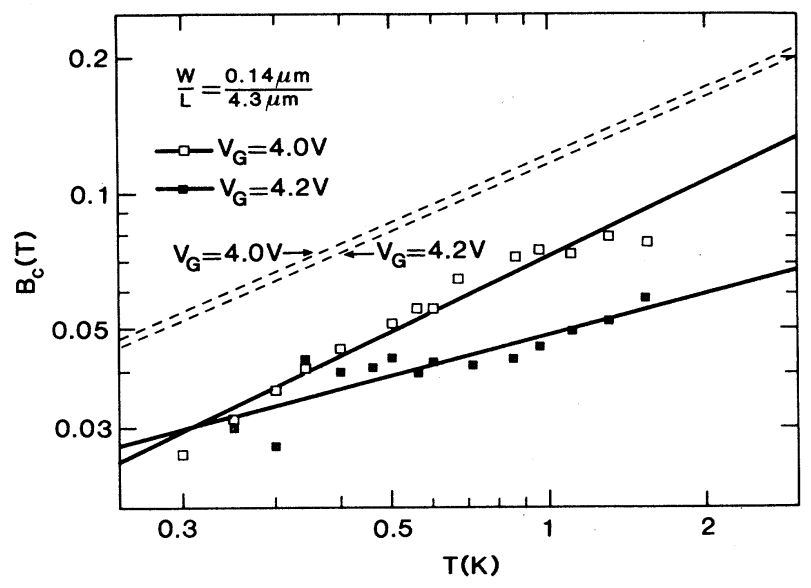

FIG. 8. Magnetic correlation length $B_{c}$ of the fluctuations as a function of temperature on a $\log -\log$ scale for $V_{G}=4.0 \mathrm{~V}$ and $V_{G}=4.2 \mathrm{~V}$. Dashed lines are corresponding theoretical relations.

$[T /(1 \mathrm{~K})]^{0.3 \pm 0.1} \mathrm{mT}$, for $V_{G}=4.0 \mathrm{~V}$ and $4.2 \mathrm{~V}$, respectively. The corresponding theoretical relations calculated using Eq. (4a) are $B_{c}^{\text {theor }}=122[T /(1 \mathrm{~K})]^{0.5} \mathrm{mT}$ and $B_{c}^{\text {theor }}=117[T /(1 \mathrm{~K})]^{0.5} \mathrm{mT}$, respectively. These relations were derived using the same assumptions for $L_{\text {in }}$ as in calculating $\mathrm{rms}(g)^{\text {theor }}$ and are displayed as dashed lines in Fig. 8. From Fig. 8 we conclude that there is a good agreement between the magnitudes of $B_{c}^{\text {expt }}$ and $B_{c}^{\text {theor }}$. Again, the slopes of the experimental lines are strongly indicative for a $T^{-1 / 2}$ behavior of $L_{\text {in }}$.

Another way of handling the data is deriving from $B_{c}$ values a set $L_{\text {in }}^{B_{c}}$ using Eq. (4a) and deriving from $\operatorname{rms}(g)$ values a set $L_{\mathrm{in}}^{\mathrm{rm}(g)}$ using Eq. (3a). Figure 9 shows these sets for $V_{G}=4.0 \mathrm{~V}$, together with $L_{\text {in }}^{\mathrm{LFMC}}$, the inelastic length derived from the low-field magnetoconductance at $1 \mathrm{~K}$ by assuming that $L_{\text {in }} \propto T^{-1 / 2}$. The general trend deduced from the figure is that $L_{\text {in }}$ derived by applying the UCF theory to our measurements exceeds $L_{\mathrm{in}}^{\mathrm{LFMC}}$. The maximum difference is roughly a factor of 2 . Some care

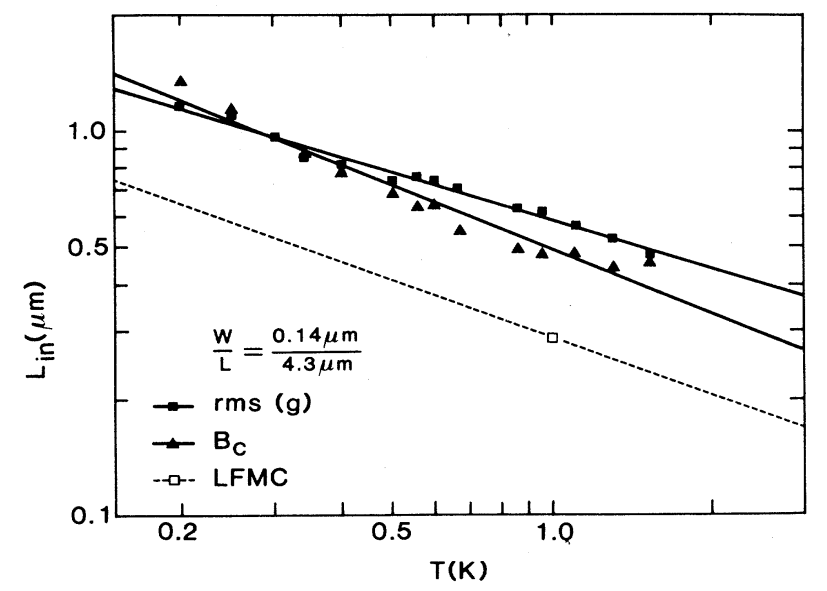

FIG. 9. Comparison of $L_{\text {in }}$ in device $N 25$ obtained from UCF and LFMC for $V_{G}=4.0 \mathrm{~V}$. $L_{\text {in }}^{\mathrm{LFMC}}$ was derived from the low-field magnetoconductance at $1 \mathrm{~K}$ by assuming $L_{\mathrm{in}} \propto T^{-1 / 2}$. should be taken, however, since $L_{\text {in }}^{\mathrm{LFMC}}$ was derived from only a single point. On the other hand, this result seems to be consistent with recent findings of Webb et al. ${ }^{14}$ who also report that in small $\mathrm{Sb}$ loops $L_{\text {in }}$ derived from UCF (using $B_{c}$ ) exceeds $L_{\text {in }}$ derived from LFMC by a factor of 2 .

In Fig. 10 traces of fluctuations in the magnetoconductance of device $N 19$ at various temperatures between 0.35 and $4.2 \mathrm{~K}$ are given. This device is relatively wide ( $W / L=0.43 \mu \mathrm{m} / 4.3 \mu \mathrm{m}$, see also Sec. IV A) and was used to study two-dimensional effects in the magnetoconductance fluctuations. The gate voltage used was $3.88 \mathrm{~V}$, giving an electron density $N_{s}$ of $1.8 \times 10^{12} \mathrm{~cm}^{-2}$ and an average conductance $\langle G\rangle$ of $106 \mu \mathrm{S}$. For the data set that includes the traces of Fig. $10 \mathrm{rms}(g)$ was calculated in the same way as for device $N 25$. The results are plotted in Fig. 11 (left-hand scale). The straight line is the best fit to the data and is given by $\operatorname{rms}(g)^{\text {expt }}=(1.5 \pm 0.3)$ $\times 10^{-2}[T /(1 \mathrm{~K})]^{-0.45 \pm 0.07}$. Further, from the experimental curves correlation functions were derived from which correlation lengths $B_{c}$ were obtained. $B_{c}$ values are also displayed in Fig. 11 (right-hand scale) and the fit is given by $B_{c}^{\text {expt }}=(58 \pm 4)[T /(1 \mathrm{~K})]^{0.23 \pm 0.1} \mathrm{mT}$. As discussed in Sec. IV A this device is two dimensional with respect to interference phenomena. For $V_{G}=3.88 \mathrm{~V}$, also using the low-field magnetoconductance experiments mentioned in Sec. IV A, it can be derived that $L_{\text {in }}=0.17$ $[T /(1 \mathrm{~K})]^{-1 / 2} \mu \mathrm{m}$ and $L_{T}=0.45[T /(1 \mathrm{~K})]^{-1 / 2} \mu \mathrm{m}$, so that the criterion $L_{\text {in }}<L_{T}<L$ is satisfied. Therefore Eqs. (3b) and (4b) are applicable. From these we find

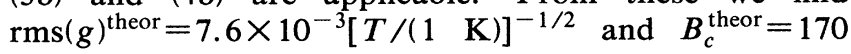
$[T /(1 \mathrm{~K})] \mathrm{mT}$. Both theoretical relations are displayed in Fig. 11 as dashed lines. We conclude that the magnitudes of $\operatorname{rms}(g)^{\text {expt }}$ and $\operatorname{rms}(g)^{\text {theor }}$ agree to within a factor of 2 , which is very satisfactory for this type of experiment. For the slope of the $\operatorname{rms}(g)$ lines the agreement is excellent, also strongly indicating a $T^{-1 / 2}$ behavior of $L_{\text {in }}$

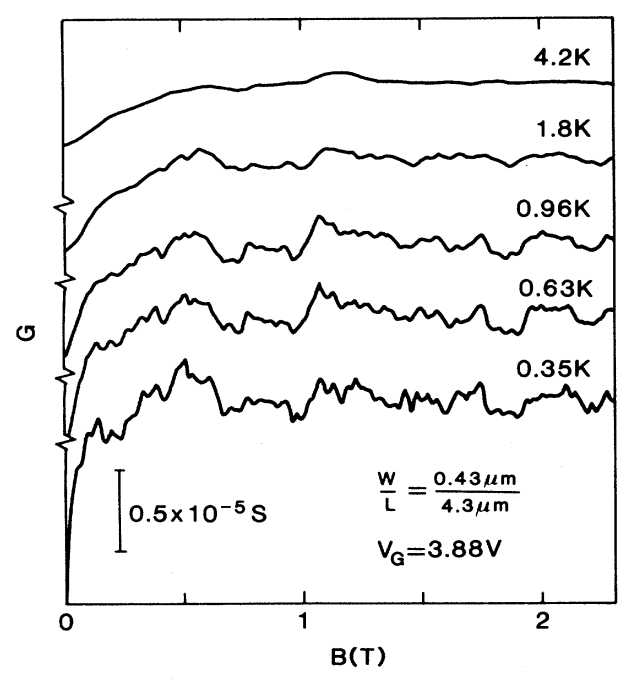

FIG. 10. Universal conductance fluctuations in the magnetoconductance of 2D device $N 19$ for a number of temperatures. $V_{G}=3.88 \mathrm{~V}$. $\langle G\rangle$ amounts to $106 \mu \mathrm{S}$. 


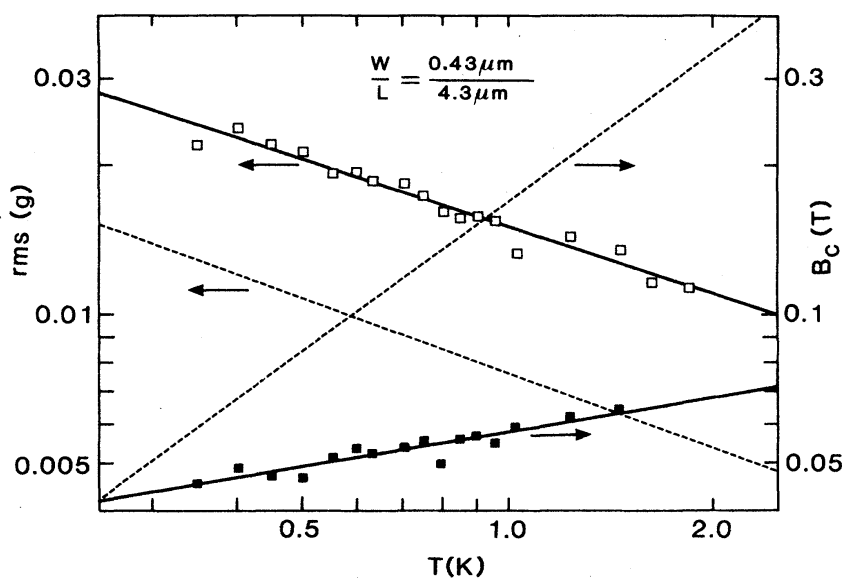

FIG. 11. Temperature dependence of $\operatorname{rms}(g)$ of the fluctuations in 2D device $N 19$ on a $\log -\log$ scale for $V_{G}=3.88 \mathrm{~V}$ (lefthand scale) and corresponding correlation lengths $B_{c}$ (righthand scale). Dashed lines are corresponding theoretical relations.

for the two-dimensional regime. For $B_{c}$ the situation is less satisfactory. Although experimental values have the proper order of magnitude, the temperature dependence of $B_{c}$ is smaller than predicted by theory. New experiments are planned in order to check whether this discrepancy is related to specific device properties.

\section{CONCLUSIONS}

We have investigated the temperature dependence of fluctuations in the magnetoconductance of narrow $\mathrm{Si}$ in- version layers in a wide temperature range $(0.2-4.2 \mathrm{~K})$ in order to check key predictions of the UCF theory. Weak-localization-effect measurements have been used for an independent determination of the inelastic diffusion length of the electrons. For the 1D geometry $\left(L_{\text {in }}>W\right.$ ) we found good agreement with the UCF theory for the magnitude of $\operatorname{rms}(g)$ and the correlation length $B_{c}$ of the fluctuations for the specified temperature range. The temperature dependence gives a strong indication that $\tau_{\text {in }}$ is proportional to $1 / T$, as expected for electronelectron scattering. For the $2 \mathrm{D}$ geometry $\left(L_{\text {in }}<W\right)$ the magnitude of $\operatorname{rms}(g)$ is in accord with the UCF theory and the temperature dependence is in excellent agreement with $\tau_{\text {in }} \propto 1 / T . \quad B_{c}$ has the proper order of magnitude, but the temperature dependence strongly deviates from the theoretical prediction. At this point new experiments are prompted.

\section{ACKNOWLEDGMENTS}

The authors wish to express their gratitude to A. Kooy and G. Boom for their advice and for MOS processing of the wafers and to L. E. M. de Groot and L. W. Lander for technical support. M. L. Horbach is acknowledged for his contributions in the starting phase of this work. We are very grateful to the Solid State Physics Group of the Faculty of Applied Physics for making available to us their dilution refrigerator and to L. J. Geerligs for technical assistance. This work is part of the research program of the Stichting Fundamenteel Onderzoek der Materie (FOM), which is financially supported by the Nederlandse Organisatie voor Wetenschappelijk Onderzoek (NWO).
${ }^{1}$ Several authors in IBM J. Res. Develop. 32, no. 3 (1988), and references therein.

${ }^{2}$ P. A. Lee and A. D. Stone, Phys. Rev. Lett. 55, 1622 (1985); P. A. Lee, A. D. Stone, and H. Fukuyama, Phys. Rev. B 35, 1039 (1987).

${ }^{3}$ J. C. Licini, D. J. Bishop, M. A. Kastner, and J. Melngailis, Phys. Rev. Lett. 55, 2987 (1985).

${ }^{4}$ S. B. Kaplan and A. Hartstein, Phys. Rev. Lett. 56, 2403 (1986).

${ }^{5}$ W. J. Skocpol, P. M. Mankiewich, R. E. Howard, L. D. Jackel, D. M. Tennant, and A. D. Stone, Phys. Rev. Lett. 56, 2865 (1986).

${ }^{6}$ S. Washburn, C. P. Umbach, R. B. Laibowitz, and R. A. Webb, Phys. Rev. B 32, 4789 (1985).

${ }^{7}$ T. J. Thornton, M. Pepper, H. Ahmed, G. J. Davies, and D. Andrews, Phys. Rev. B 36, 4514 (1987).

${ }^{8}$ J. R. Gao, J. Caro, A. H. Verbruggen, S. Radelaar, and J. Mid- delhoek, Microelectron. Eng. 9, 373 (1989).

${ }^{9}$ B. L. Al'tshuler and A. G. Aronov, Pis'ma Zh. Eksp. Teor. Fiz. 33, 515 (1981) [JETP Lett. 33, 499 (1981)]

${ }^{10}$ D. J. Bishop, R. C. Dynes, and D. C. Tsui, Phys. Rev. B 26, 773 (1982).

${ }^{11}$ K. F. Berggren, T. J. Thornton, D. J. Newson, and M. Pepper, Phys. Rev. Lett. 57, 1769 (1986).

${ }^{12}$ W. J. Skocpol, L. D. Jackel, E. L. Hu, R. E. Howard, and L. A. Fetter, Phys. Rev. Lett. 49, 951 (1982).

${ }^{13}$ P. A. Lee and T. V. Ramakrishnan, Rev. Mod. Phys. 57, 287 (1985).

${ }^{14}$ R. A. Webb, S. Washburn, H. J. Haucke, and A. D. Benoit, in Proceedings of the Fifth International Winter School on Physics and Technology of Submicron Structures, Mauterndorf, Austria, edited by H. Heinrich, G. Bauer, and F. Kuchar (Springer, New York, 1988), p. 98. 